\title{
Le déficit immunitaire commun variable: un modèle de maladie liée à un déficit du mécanisme d'hypermutation somatique des gènes des immunoglobulines
}

\section{$\mathbf{L}$} e déficit immunitaire commun variable (DICV) est un syndrome hétérogène caractérisé par une hypogammaglobulinémie touchant tous les isotypes d'immunoglobulines, des infections bactériennes à répétition, et une incidence augmentée de manifestations auto-immunes ou de tumeurs (revue dans [1]). Le diagnostic est fondé sur l'exclusion des autres causes connues de déficit immunitaire en anticorps. La transmission génétique est autosomique récessive, autosomique dominante ou liée à l'X. Cependant, les cas sporadiques sont les plus fréquents. Le DICV touche les hommes et les femmes de manière équivalente et peut être diagnostiqué à n'importe quel âge de la vie. Cela contraste avec l'hypogammaglobulinémie liée à l'X où les infections récurrentes apparaissent dans les deux premières années de la vie.

Un tableau clinique d'immunodéficience

Les manifestations cliniques liées du DICV sont marquées par des infections répétées à germes encapsulés des voies aériennes supérieures. Les épisodes septicémiques, les infections répétées de la peau, du tractus urinaire, des articulations et du système nerveux central peuvent également être notés mais sont beaucoup plus rares. Certains patients développent des infections inhabituelles pour un déficit humoral comme des infections à mycobactéries, mycotiques, parasitaires ou à germes opportunistes comme le Pneumocystis carinii. Les infections répétées à Herpes sim- plex sont fréquentes et touchent environ $50 \%$ des patients. Les infections digestives à Lamblia, ou à des bactéries pathogènes sont également fréquentes. Le traitement du DICV repose sur l'administration régulière par voie intraveineuse d'immunoglobulines polyvalentes. La décision de traiter est liée à la fréquence et la sévérité des infections bactériennes. Les mécanismes de l'hypogammaglobulinémie du DICV ne sont pas connus. Le nombre de lymphocytes B circulants est normal ou augmenté chez ces malades. Leur phénotype est celui des cellules B mûres : CD19+, $\mathrm{CD} 20^{+}, \mathrm{CD} 10^{-}$. Les différentes études réalisées ne permettent pas de conclure formellement en faveur d'un déficit intrinsèque cellulaire $B$ ou T. Selon les auteurs, la production quantitative d'anticorps par les lymphocytes B après activation in vitro peut être altérée ou non. Il n'existe pas d'anomalies de l'activation des lymphocytes B ou de la commutation isotypique. Cependant, les résultats des études fonctionnelles in vitro sont, le plus souvent, difficiles à interpréter en raison de l'hétérogénéité des malades étudiés, du contexte d'infections chroniques ou du traitement des patients par perfusions régulières d'immunoglobulines intraveineuses.

Malgré ces difficultés, l'observation des deux faits cliniques suivants nous a amenés à aborder la physiopathologie du DICV par une nouvelle approche : d'une part, la survenue d'infections sévères et répétées chez certains patients ayant des concentrations d'IgG, d'IgA et d'IgM sériques paradoxalement non effondrées et, d'autre part, la nécessité de débuter le traitement substitutif par perfusion d'immunoglobulines chez certains patients ayant une hypogammaglobulinémie globale modérée mais atteints d'infections bactériennes récurrentes [1]. Ces observations évoquent la possibilité d'une anomalie qualitative des anticorps produits par les lymphocytes B, associée au déficit quantitatif en immunoglobulines habituellement constaté dans le DICV.

\section{L'hypermutation somatique}

Le mécanisme d'hypermutation somatique des gènes codant pour les régions variables des immunoglobulines $(\mathrm{IgV})$ est un processus impliqué dans la diversification du répertoire permettant d'augmenter l'affinité d'un anticorps pour un antigène [2]. Ce processus intervient dans le centre germinatif des organes lymphoïdes secondaires lors des réponses immunitaires aux antigènes dépendantes des lymphocytes T [3]. Les centres germinatifs sont formés à partir de l'expansion clonale de quelques cellules B naïves sélectionnées lors de la réponse immunitaire primaire au niveau de la zone $\mathrm{T}$ des ganglions. Initialement, les lymphocytes B prolifèrent dans la zone sombre des centres germinatifs et subissent le processus de mutation somatique des gènes codant pour les régions variables des chaînes lourdes $(V H)$ et légères $(V L)$. La majorité de ces cellules B meurent par un phénomène d'apoptose. Les lymphocytes B exprimant un récepteur pour l'antigène avec une affinité augmentée 
sont sélectionnés par l'antigène présenté par les cellules dendritiques folliculaires et par l'intervention de molécules exprimées par les cellules T au niveau des zones claires du centre germinatif. Sous l'influence de molécules costimulatrices et de différentes cytokines, les lymphocytes B sélectionnés se différencient en plasmocytes ou en lymphocytes B mémoires qui quittent les organes lymphoïdes secondaires pour la périphérie [4]. Les lymphocytes B mémoires sont définis chez l'homme par l'expression des antigènes CD19, CD20 et d'un isotype secondaire d'immunoglobuline, principalement IgG ou IgA.

Plusieurs travaux récemment publiés se sont intéressés à la répartition des cellules B naïves et mémoires du sang chez l'homme, au répertoire des gènes $V H$ et $V L$ exprimés par ces cellules et à la fréquence des mutations somatiques de ces gènes. L'ensemble de ces travaux montrent que les lymphocytes B naïs (CD19+/ $\mathrm{IgM}^{+} /$ $\left.\mathrm{IgD}^{+}\right)$et mémoires $\left(\mathrm{C}_{1} 9^{+} / \mathrm{IgM}^{-} /\right.$ $\left.\mathrm{IgD}^{-}\right)$représentent respectivement environ $75 \%$ et $15 \%$ des cellules B CD19; $10 \%$ des cellules B périphériques expriment le phénotype CD19+/IgM+/IgD-. Le taux des mutations somatiques des gènes $V H$ et $V L$ exprimées par ces différentes populations de lymphocytes B est d'environ $0,2 \%$ pour les cellules B naïves, et de $3,9 \%$ à $4,2 \%$ pour les cellules B mémoires [5-7].

\section{Une hypomutation somatique des gènes des immunoglobulines s'accompagne d'immunodéficience}

Nous avons étudié la fréquence des mutations somatiques des gènes des immunoglobulines des lymphocytes B $\mathrm{du}$ sang de huit patients (quatre adultes et quatre enfants) ayant un DICV [8]. Deux approches complémentaires ont été utilisées dans cette étude: (1) l'analyse des séquences introniques JH4-JH5 situées en position 3' des réarrangements $\mathrm{VH}-\mathrm{JH} 4$ à partir de lymphocytes B mémoires $\left(\mathrm{CD}^{+}{ }^{+} \mathrm{IgM}^{-} \mathrm{IgD}^{-}\right)$purifiés du sang, et (2) l'analyse directe des transcrits V3-23-Cy isolés à partir des cellules mononucléées du sang. La première

\begin{tabular}{|lcc|}
\hline \multicolumn{2}{c|}{ Tableau I } \\
\multicolumn{1}{c|}{ MUTATIONS DES TRANSCRITS V3-23-C $\gamma$} \\
\hline \multicolumn{1}{c|}{ Clones mutés (\%) } & Mutation/pb (\%) \\
\hline Témoins & & \\
1 & 100 & 7,9 \\
2 & 100 & 7,1 \\
Patients & & \\
1 & 25 & 0,8 \\
2 & 60 & 1,6 \\
3 & 100 & 5,1 \\
4 & 100 & 5,4 \\
5 & 86 & 5,4 \\
6 & 100 & 6,2 \\
\hline
\end{tabular}

«l'empreinte» du mécanisme d'hypermutation somatique au niveau de séquences génomiques non sélectionnées. Le fait que le gène JH4 soit utilisé par la moitié des réarrangements des lymphocytes B permet l'étude d'un grand nombre de lymphocytes B évitant l'analyse d'un gène $V H$ unique. En revanche, la seconde approche nous a permis d'étudier directement les transcrits Cy des lymphocytes B mémoires utilisant un gène $V H$ sélectionné : le gène V3-23, membre de la famille VH3 utilisé par environ 4-10\% des lymphocytes B. Les résultats obtenus ont permis tout d'abord de montrer que le mécanisme d'hypermutation somatique s'étendait bien au-delà du réarrangement $\mathrm{VDJH}$, au niveau de l'intron JH, comme cela avait été décrit chez d'autres espèces. Par ailleurs, les deux approches utilisées ont été concordantes puisque la majorité des séquences introniques et des transcrits V3-23-C $\gamma$ analysés chez les sujets normaux était fortement mutée (en moyenne le taux de mutation est de $3,6 \%$ pour les séquences introniques et de $7,5 \%$ pour les séquences V3-23-C $\gamma$ ) (Tableau I). Dans six cas de DICV étudiés, l'ensemble des séquences analysées, quelle que soit l'approche, présente un taux de mutation variant entre $65 \%$ à $80 \%$ de celui des sujets normaux. De manière intéressante, dans deux cas, on a trouvé une diminution significative de la fréquence des mutations avec un taux de $0,8 \%$ et de $1,6 \%$ de mutation au niveau du gène $V 3-23$. En outre, dans ces deux cas, $40 \%$ à $75 \%$ des séquences issues des lymphocytes B IgG du sang sont totalement dépourvues de mutation, et expriment un gène germinal $V 3-23$. A notre connaissance, on n'a jamais mis en évidence de compartiment de cellules B mémoires totalement dépourvu de mutation somatique.

\section{Un défaut spécifique des cellules $B$ ?}

L'étude phénotypique et fonctionnelle des lymphocytes $\mathrm{T}$ de ces deux patients n'a pas montré d'anomalie évidente (activation en présence de mitogènes, production de cytokines, expression du ligand du CD40). Enfin, on a étudié les qualités de la fonction auxiliaire des lymphocytes $\mathrm{T}$ de ces patients par un test d'induction in vitro des mutations somatiques. En effet, il a été montré récemment [10] que la stimulation in vitro de la lignée de lymphome de Burkitt BL2 en présence d'anticorps anti-IgM et d'un clone lymphocytaire $\mathrm{T}$ induit l'accumulation de mutations somatiques au niveau du gène $V H$ réarrangé de cette lignée. Nous avons pu adapter ce système de culture par l'utilisation des lymphocytes $\mathrm{T}$ des patients activés in vitro. Ces résultats ont permis de montrer qu'à des degrés variables, les lymphocytes $\mathrm{T}$ de ces deux patients étaient capables de délivrer des signaux permettant l'induction de mutations somatiques au niveau des gènes $V H$ de la lignée BL2. L'ensemble de ces résultats suggère que le déficit des mutations somatiques observé chez ces patients est lié à un défaut spécifique des cellules 
B plus qu'à une anomalie fonctionnelle des lymphocytes $\mathrm{T}$ ou à un défaut de collaboration entre les lymphocytes $\mathrm{T}$ et les lymphocytes $\mathrm{B}$.

Ces résultats pourraient avoir plusieurs implications tant dans la compréhension de la physiopathologie du DICV que dans l'étude du mécanisme des mutations somatiques des gènes $V$ des immunoglobulines. Tout d'abord, l'étude de la fréquence de ce type d'anomalie sur une plus grande cohorte de patients est nécessaire. Cela devrait permettre de distinguer, au sein du syndrome hétérogène qu'est le DICV caractérisé par un défaut quantitatif, variable, de la production d'anticorps, des patients ayant une fréquence normale, ou discrètement diminuée, des mutations somatiques des gènes $V$, d'un second groupe de patients caractérisé par un défaut plus sévère de ce processus. Par ailleurs, le mécanisme moléculaire de l'hypermutation somatique des gènes $V$ est actuellement inconnu, vraisemblablement en raison de sa complexité mais également par l'absence de modèles animaux ou, jusqu'à récemment, de tests cellulaires reproductibles. Chez ces patients, les mécanismes moléculaires permettant la mise en œuvre des mécanismes des mutations somatiques, ou les étapes de transmission de signaux condui- sant à l'activation de ce processus, pourraient être altérés. L'étude de différentes voies de transmission du signal à partir du récepteur B des lymphocytes de ces patients pourrait permettre d'approcher certains mécanismes impliqués dans la cascade de signalisation aboutissant aux mutations somatiques.

Enfin, on ne peut pas exclure que l'anomalie constatée soit secondaire, et non initialement responsable du déficit humoral. Cependant, ces patients ont un répertoire de lymphocytes B mémoires biaisé par l'expression de gènes $V H$ non mutés. Cette anomalie peut contribuer, en association avec le défaut de production des anticorps, à aggraver le déficit immunitaire. Sur le plan clinique, ces résultats suggèrent que les perfusions d'immunoglobulines chez ces patients doivent être adaptées à la sévérité et à la fréquence des infections et non à la concentration sérique des anticorps

\section{Yves Lévy}

Service d'immunologie clinique, CHU Henri-Mondor, 51, avenue du Maréchalde-Lattre-de-Tassigny, 94010 Créteil Cedex, France.

\section{TIRÉS À PART}

Y. Lévy.

\section{RÉFÉRENCES}

1. Sneller MC, Strober W, Eisenstein E, Jaffe JS, Cunningham-Rundles C. NIH conference: new insights into common variable immunodeficiency. Ann Intern Med 1993 ; $188: 720-30$.

2. Neuberger MS, Milstein C. Somatic hypermutation. Curr Opin Immunol $1995 ; 7$ : 248-54.

3. Berek C, Ziegner M. The maturation of the immune response. Immunol Today 1993 ; $14: 400-4$.

4. Liu YJ, Arpin C. Germinal center development. Immunol Rev 1997 ; 156 : 111-126.

5. Wagner SD, Elvin JG, Norris P, McGregor JM, Neuberger MS. Somatic hypermutation of Ig genes in patients with xeroderma pigmentosum (XP-D). Int Immunol 1996 ; $85^{\circ}$ : 701-5.

6. Klein U, Küppers R, Rajewsky K. Human IgM+ IgD+ B cells, the major B cell subset in the peripheral blood, express Vk genes with no or little somatic mutation throughout life. Eur J Immunol 1993 ; 23 : 3272-7.

7. Klein U, Küppers R, Rajewsky K. Evidence for a large compartment of IgMexpressing memory B cells in humans. Blood 1997 ; 89 : 1288-98.

8. Levy Y, Gupta N, Le Deist F, Fischer A, Weill JC, Reynaud CA. Defect gene in $I G V$ gene somatic hypermutation in common variable immuno-deficiency syndrome. Proc Natl Acad Sci USA 1998 ; 95 : 13135-40.

9. Denépoux S, Razanajoana D, Blanchard D, Meffre G, Capra JD, Banchereau J, Lebecque $\mathrm{S}$. Induction of somatic mutation in a human B cell line in vitro. Immunity $1997 ; 6: 35-46$

\section{.}

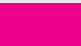

.

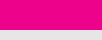

政 